\title{
Determinación de los efectos parásitos capacitivos e inductivos, debidos a las pistas de una tarjeta de circuito impreso cuando se utiliza un filtro $\pi$
}

\author{
Capacitive and Inductive Parasitic Effects Determination, \\ Due to the Traces in a Printed Circuit Board, when Using a $\pi$ Type Filter
}

\author{
Uribe-Cruz O. \\ Escuela Superior de Ingeniería Mecánica y Eléctrica \\ Unidad Zacatenco del Instituto Politécnico Nacional \\ E-mail:omar_uribe@hotmail.com
}

\author{
Peña-Rivero R. \\ Escuela Superior de Ingeniería Mecánica y Eléctrica \\ Unidad Zacatenco del Instituto Politécnico Nacional \\ E-mail:rpenar@ipn.mx
}

Información del artículo: recibido: junio de 2008, reevaluado: marzo de 2010, aceptado: febrero de 2011

\section{Resumen}

En este trabajo se presenta el cálculo de los efectos parásitos capacitivos e inductivos debidos a las pistas de una tarjeta de circuito impreso. Para encontrar sus valores y sus efectos, se desarrolló un programa en MatLab, validando los resultados con la solución del circuito eléctrico equivalente utilizando el programa comercial Serenade. En este trabajo de investigación se utilizó un filtro tipo $\pi$ de segundo orden, el cual se implementó en una tarjeta de circuito impreso comercial de una sola cara.

\section{Abstract}

In this work the parasitic capacitive and inductive effects due to the strip lines in a printed circuit board are calculated. To find their values a MatLab code program was implemented and validated solving the electric equivalent circuit using the commercial software called Serenade. In this research a second order $\pi$-type filter was used in a commercial single-layer printed circuit board.

\section{Descriptores}

- capacitancia mutua

- inductancia mutua

- efectos parásitos capacitivos e inductivos

- microcinta

- tarjeta de circuito impreso

\section{Keywords}

- mutual capacitance

- mutual inductance

- capacitive and inductive parasitic effects

- microstrip

- printed circuit board 


\section{Introducción}

En el diseño de los sistemas electrónicos digitales, suelen emplearse diferentes tipos de filtros en la línea de alimentación para reducir el ruido de alta frecuencia que se genera debido a las propias conmutaciones que se presentan en el sistema. El filtro pasa-bajas más comúnmente usado, debido a su bajo costo y fácil implementación, es el que utiliza un capacitor, el cual es efectivo hasta unos cuantos MegaHerz (Zeeff et al., 2003). Sin embargo, la corriente que fluye a través del capacitor acopla magnéticamente la malla que suele formarse de un lado del filtro con la que se forma del otro lado. Este acoplamiento magnético tiene mayor impacto a altas frecuencias haciendo que la efectividad del filtro se vea reducida (Zeeff et al., 2003). En este artículo se presentan los resultados obtenidos al calcular los efectos parásitos capacitivos e inductivos que se presentan en un filtro $\pi$ de segundo orden, en el cual se puede claramente observar los efectos de las capacitancias e inductancias mutuas que se presentan, cuando se utilizan este tipo de filtros.

\section{Desarrollo}

Capacitancia mutua entre pistas paralelas

La capacitancia mutua entre pistas paralelas en una tarjeta de circuito impreso se calcula por (Gupta et al., 1996):

$$
C_{m}\left(\varepsilon_{r}\right)=\frac{1}{2}\left[C_{i m p a r}\left(\varepsilon_{r}\right)-C_{p a r}\left(\varepsilon_{r}\right)\right]
$$

para el modo impar, la capacitancia se calcula con la siguiente expresión:

$$
C_{\text {impar }}\left(\varepsilon_{r}\right)=C_{p}+C_{f}+C_{f^{\prime}}
$$

donde

$$
\begin{aligned}
& C_{p}=\frac{\varepsilon_{r 0} \varepsilon_{r}}{h} \\
& C_{f}=\frac{1}{2}\left[\frac{\sqrt{\varepsilon_{r}}}{c Z_{0}}-C_{p}\right] \\
& C_{f}=\frac{C_{f}^{4} \sqrt{\frac{\varepsilon_{r 0}}{\varepsilon_{r e}}}}{1+A\left(\frac{h}{d}\right) \tan h\left(\frac{10 a}{h}\right)}
\end{aligned}
$$

$$
A=e^{-0.1 e^{2.33-\frac{1.5 a}{h}}}
$$

en nuestro caso:

$$
\begin{aligned}
& Z_{0}=\frac{60}{\sqrt{\varepsilon_{r e}}} \ln \left(\frac{8 h}{a}+0.25 \frac{a}{h}\right) \\
& \text { dado que } \frac{a}{h} \leq 1 \\
& \varepsilon_{r e}=\frac{\varepsilon_{r}+1}{2}+\frac{\varepsilon_{r}-1}{2} \sqrt{1+\frac{10 h}{a}}
\end{aligned}
$$

donde $C_{p^{\prime}} C_{f^{\prime}} C_{f^{\prime}}$, representan las capacitancias de placas paralelas y las capacitancias de los perfiles de las pistas que forman la estructura. El cálculo de la capacitancia mutua se obtiene sustituyendo los valores de las constantes que se pueden observar en la figura 1.

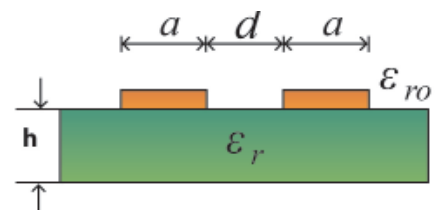

Figura 1. Tarjeta de circuito impreso con dos pistas paralelas

Cálculo de la inductancia de un conductor delgado de geometría rectangular

La inductancia de un conductor de sección rectangular como se muestra en la figura 2 , se puede calcular utilizando la siguiente ecuación (9):

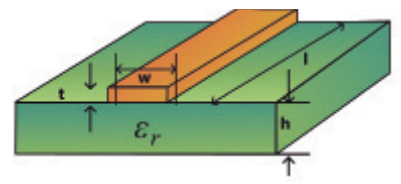

Figura. 2. Pista de cobre en Tarjeta de circuito Impreso

$$
L=0.002 l\left(\ln \left(\frac{2 l}{w+t}\right)+0.50049+\frac{w+t}{3 l}\right)[\mu H]
$$

donde

$w=$ ancho de la pista,

$t$ = grosor de la pista de cobre,

$l$ = longitud del conductor.

Las unidades están dadas en $\mathrm{cm}$. 
Inductancia de una estructura espiral plana de $n$ vueltas

La inductancia de la estructura plana se calcula con Uribe (2005), considerando un inductor recto como se muestra en la figura 3. La inductancia total de un conductor es la suma de las inductancias propias y las inductancias mutuas:

$L_{T}=L_{O}-M_{+}-M_{-}$

donde

$L_{T}=$ inductancia total,

$L_{O}=$ suma de las inductancias propias de los segmentos rectos,

$M_{+}=$suma de las inductancias mutuas positivas,

$M_{-}=$suma de las inductancias mutuas negativas.

Inductancia mutua entre pistas paralelas

La inductancia mutua entre dos conductores paralelos está dada en función de la longitud de los mismos y de la distancia geométrica que existe entre ellos. La inductancia mutua de dos conductores se calcula por:

$M=2 l F$

donde $l$ es la longitud del conductor en centímetros. $F$ es el parámetro de la inductancia mutua, el cual se puede calcular utilizando la siguiente fórmula:

$F=\ln \left(\frac{l}{d}+\left[1+\left(\frac{l}{d}\right)^{2}\right]^{\frac{1}{2}}\right)-\left[1+\left(\frac{d}{l}\right)^{2}\right]^{\frac{1}{2}}+\left(\frac{d}{l}\right)$

donde $d$ es la distancia geométrica entre dos conductores, que es aproximadamente igual a la distancia que existe entre el centro de los conductores.

Considerando los segmentos de los conductores mostrados en la figura 3,

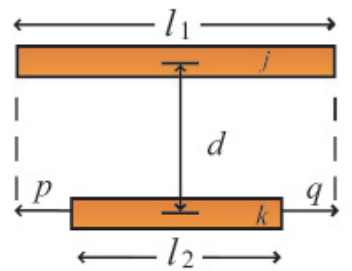

Figura 3. Segmento de dos conductores para el cálculo de la inductancia mutua $j \mathrm{y} k$ corresponden a los índices del conductor, $p$ y $q$ a los índices de la longitud que complementa la diferencia de un conductor respecto al otro. La inductancia mutua de los conductores $j$ y $k$ en la configuración de la figura 3 es:

$M_{j, k}=\frac{1}{2}\left\{\left(M_{k+p}+M_{k+q}\right)-\left(M_{p}+M_{q}\right)\right\}$

$\mathrm{M}_{j, k}=\frac{1}{2}\left\{\left(M_{k}+M_{k+q}\right)-M_{q}\right\} \quad$ para $p=0$

$\mathrm{M}_{j, k}=\frac{1}{2}\left\{\left(M_{k+p}+M_{k}\right)-M_{p}\right\} \quad$ para $q=0$

$\mathrm{M}_{j, k}=M_{k+p}-M_{p}$

para $p=q$

$\mathrm{M}_{j, k}=M_{k}$

para $p=q=0$

Si la longitud de $l_{1}$ y $l_{2}$ son las mismas $\left(l_{1}=l_{2}\right)$, se utiliza la ecuación (13d). Cada término de inductancia mutua en la ecuación 13 se calcula usando las ecuaciones 11 y 12.

$M_{k+p}=2 l_{k+p} F_{k+p}$

donde

$$
\begin{gathered}
F_{k+p}=\ln \left\{\left(\frac{l_{k+p}}{d_{j, k}}\right)+\left[1+\left(\frac{l_{k+p}}{d_{j, k}}\right)^{2}\right]^{\frac{1}{2}}\right\}- \\
{\left[1+\left(\frac{d_{j, k}}{l_{k+p}}\right)^{2}\right]^{\frac{1}{2}}+\left(\frac{d_{j, k}}{l_{k+p}}\right)}
\end{gathered}
$$

Determinación matemática de las inductancias propias y mutuas debido a la geometría utilizada en el filtro RC

En términos de las ecuaciones (3-9) se desglosaron las fórmulas para el cálculo de las inductancias propias y mutuas, debido a la geometría que forma el filtro construido en una tarjeta de circuito impreso, la cual se muestra en la figura 4 . De la ecuación 3, sabemos que se tiene que calcular cada inductancia propia, debido a cada segmento que forma un trazo de cada pista, para cada trazo se calculó la inductancia propia. Por lo tanto, para la malla 1 la inductancia propia se tiene: 
$I_{m 1}=I_{l 2}+I_{l 2}+2 * l_{l 5}$

donde

$I_{l 2}=$ inductancia propia de la pista de longitud $l_{2}$

$I_{l 2^{\prime}}=$ inductancia propia de la pista de longitud $l_{2^{\prime}}{ }^{\prime}$

$I_{l 5}=$ inductancia propia de la pista de longitud $l_{5}$,

Para la inductancia propia de la malla 2:

$I_{m 2}=I_{l 1}+I_{l 1^{\prime}}+2 *\left(l_{l 2}+l_{l 2^{\prime}}\right)+I_{l 3}$

donde

$I_{l 1} \quad$ inductancia propia de la pista de longitud $l_{1}$

$I_{l 1^{\prime}}=$ inductancia propia de la pista de longitud $l_{1^{\prime}}{ }^{\prime}$

$I_{l 2}=$ inductancia propia de la pista de longitud $l_{2}{ }^{\prime}$

$I_{l 2^{\prime}}=$ inductancia propia de la pista de longitud. $l_{2^{\prime}}$,

$I_{l 3}=$ Inductancia propia de la pista de longitud $l_{3}$.

$l_{3}=l_{1}+l_{1}+g a p$

gap = separación entre pistas (localización de $R_{1}$ ).

Para la inductancia propia de la malla 3:

$I_{m 1}=I_{l 2}+I_{l 2^{\prime}}+2 * l_{l 6}$

donde

$I_{12}=$ inductancia propia de la pista de longitud $l_{2}$,

$I_{l 2^{\prime}}=$ inductancia propia de la pista de longitud $l_{2^{\prime}}$,

$I_{l 6}=$ inductancia propia de la pista de longitud $l_{6}$.

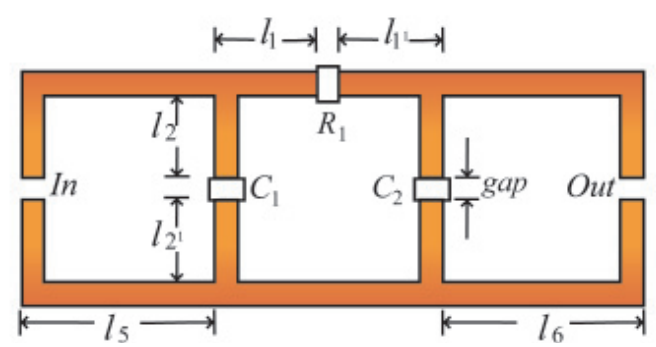

Figura 4. Geometría utilizada en la construcción de las tarjetas de circuito impreso para la implementación del filtro RC

Las inductancias mutuas negativas para el circuito de la figura 4 se resuelven utilizando las siguientes expresiones:

$M_{\text {uno }}=2 *\left(M_{5,5}+M_{2,4^{\prime}}+M_{2,4}\right)$
$M_{\text {dos }}=2 *\left(M_{1,3}+M_{1,3^{\prime}}+M_{2,4}+M_{2,4^{\prime}}\right)$

$M_{\text {tres }}=2 *\left(M_{6,6}+M_{2,4^{\prime}}+M_{2,4}\right)$

donde

$M_{j k}=$ inductancia mutua entre el conductor $j$ y el conductor $k$

$M_{13}=1 / 2\left(M_{3}+M_{1}-M_{1 \text { gap }^{\prime}}\right)$

$M_{13^{\prime}}=1 / 2\left(M_{3}+M_{1^{\prime}}-\right.$,

$M_{24}=1 / 2\left(M_{4}+M_{2}-M_{2 g_{a p^{\prime}}}-M_{4 g_{a p^{\prime}}}\right)$

$M_{24^{\prime}}=1 / 2\left(M_{4}+M_{2^{\prime}}-M_{2 \text { gap }}-M_{4 \text { gap }}\right)$

donde

$M_{1}=2 * l_{1} * F_{1}$

$M_{1^{\prime}}=2 * l_{1^{\prime}} * F_{1}$

$M_{2}=2 * l_{2} * F_{2}$

$M_{2^{\prime}}=2 * l_{2^{\prime}} * F_{2}$

$M_{3^{\prime}}=2 * l_{3^{\prime}} * F_{3}$

$M_{4}=2 * l_{4} * F_{4}$

$M_{4^{\prime}}=2 * l_{4^{\prime}} * F_{4}$

$M_{5^{\prime}}=2 * l_{5} * F_{5}$

$M_{6^{\prime}}=2 * l_{6} * F_{6}$

$M_{1+\text { gap }}=2 * l_{1+\text { gap }} * F_{1+\text { gap }}$

$M_{1^{\prime}+\text { gap }}=2 * l_{1^{\prime}+\text { gap }} * F_{1^{\prime}+\text { gap }}$

$M_{2+\text { gap }}=2 * l_{2+\text { gap }} * F_{2+\text { gap }}$

$M_{2^{\prime}+\text { gap }}=2 * l_{2^{\prime}+\text { gap }} * F_{2^{\prime}+\text { gap }}$

$M_{4+\text { gap }}=2 * l_{4+\text { gap }} * F_{4+\text { gap }}$

$M_{4^{\prime}+\text { gap }}=2 * l_{4^{\prime}+\text { gap }} * F_{4^{\prime}+\text { gap }}$

donde

$F_{k} \quad$ es el parámetro de la inductancia mutua del conductor $k$ 
$F_{1}=\ln \left(\frac{l_{1}}{d_{1,3}}+\left[1+\left(\frac{l_{1}}{d_{1,3}}\right)^{2}\right]^{\frac{1}{2}}\right)-\left(1+\left(\frac{d_{1,3}}{l_{1}}\right)^{2}\right)^{\frac{1}{2}}+\frac{d_{1,3}}{l_{1}}$

$F_{1^{\prime}+g q p}=\ln \left(\frac{l_{1^{\prime}+g a p}}{d_{1^{\prime}+g p, 3}}+\left[1+\left(\frac{l_{1^{\prime}+g a p}}{d_{1^{\prime}+g q p, 3}}\right)^{2}\right]^{2}\right]^{\frac{1}{2}}+\frac{d_{1^{\prime}, 3}}{l_{1^{\prime}}}-\left(1+\left(\frac{d_{1^{\prime}+g p, 3}}{l_{1^{\prime}+g a p}}\right)^{2}\right)^{\frac{1}{2}}$

$F_{1+g a p}=\ln \left(\frac{l_{1+g a p}}{d_{1+g a p, 3}}+\left[1+\left(\frac{l_{1+g a p}}{d_{1+g a p, 3}}\right)^{2}\right]^{\frac{1}{2}}\right)+\frac{d_{1,3}}{l_{1}}-\left(1+\left(\frac{d_{1+g a p, 3}}{l_{1+g a p}}\right)^{2}\right)^{\frac{1}{2}}$

$F_{2}=\ln \left(\frac{l_{2}}{d_{2,4}}+\left[1+\left(\frac{l_{2}}{d_{2,4}}\right)^{2}\right]^{\frac{1}{2}}\right)-\left(1+\left(\frac{d_{2,4}}{l_{2}}\right)^{2}\right)^{\frac{1}{2}}+\frac{d_{2,4}}{l_{2}}$

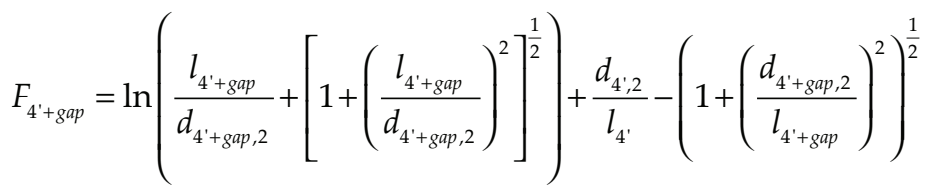

$F_{4+g q p}=\ln \left(\frac{l_{4+g q p}}{d_{4+g a p, 2}}+\left[1+\left(\frac{l_{4+g a p}}{d_{4+g q p, 2}}\right)^{2}\right]^{\frac{1}{2}}\right)+\frac{d_{4,2}}{l_{4}}-\left(1+\left(\frac{d_{4+g a p, 2}}{l_{4+g a p}}\right)^{2}\right)^{\frac{1}{2}}$

$F_{3}=\ln \left(\frac{l_{3}}{d_{1,3}}+\left[1+\left(\frac{l_{3}}{d_{1,3}}\right)^{2}\right]^{\frac{1}{2}}\right)-\left(1+\left(\frac{d_{1,3}}{l_{3}}\right)^{2}\right)^{\frac{1}{2}}+\frac{d_{1,3}}{l_{1}}$

$F_{4}=\ln \left(\frac{l_{4}}{d_{4,2}}+\left[1+\left(\frac{l_{4}}{d_{4,2}}\right)^{2}\right]^{\frac{1}{2}}\right)-\left(1+\left(\frac{d_{4,2}}{l_{4}}\right)^{2}\right)^{\frac{1}{2}}+\frac{d_{4,2}}{l_{4}}$

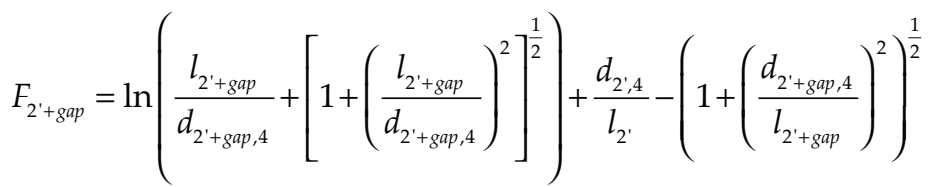

$F_{2+g a p}=\ln \left(\frac{l_{2+8 a p}}{d_{2+g a p, 4}}+\left[1+\left(\frac{l_{2+g a p}}{d_{2+g a p, 4}}\right)^{2}\right]^{\frac{1}{2}}\right)+\frac{d_{2,4}}{l_{2}}-\left(1+\left(\frac{d_{2+g a p, 4}}{l_{2+g a p}}\right)^{2}\right)^{\frac{1}{2}}$ 


$$
\begin{aligned}
& F_{6}=\ln \left(\frac{l_{6}}{d_{6,6}}+\left[1+\left(\frac{l_{6}}{d_{6,6}}\right)^{2}\right]^{\frac{1}{2}}\right)-\left(1+\left(\frac{d_{6,6}}{l_{6}}\right)^{2}\right)^{\frac{1}{2}}+\frac{d_{6,6}}{l_{6}} \\
& F_{5}=\ln \left(\frac{l_{5}}{d_{5,5}}+\left[1+\left(\frac{l_{5}}{d_{5,5}}\right)^{2}\right]^{\frac{1}{2}}\right)-\left(1+\left(\frac{d_{5,5}}{l_{5}}\right)^{2}\right)^{\frac{1}{2}}+\frac{d_{5,5}}{l_{5}}
\end{aligned}
$$

donde

$$
\begin{aligned}
& M_{\text {uno }}=\text { inductancia mutua que producen las pistas en } \\
& \text { la malla } 1 \text { del circuito, } \\
& M_{d o s}=\text { inductancia mutua que producen las pistas en } \\
& \text { la malla } 2 \text { del circuito, } \\
& M_{\text {tres }}=\text { inductancia mutua que producen las pistas en } \\
& \text { la malla } 3 \text { del circuito, } \\
& M_{x, y}=\text { inductancia mutua que se produce entre la pis- } \\
& \text { ta } l_{x} \text { y la pista } l_{y} \text {, } \\
& d_{x, y}=\text { distancia entre los centros de los conductores } \\
& l_{x} \text { y } l_{y} \text {, } \\
& F_{x} \quad=\text { parámetro de la inductancia mutua vista desde } \\
& \text { el conductor de longitud } l_{x} \text {. }
\end{aligned}
$$

Circuito ideal y equivalente eléctrico del Filtro RC de segundo orden propuesto y su función de transferencia

Para demostrar y cuantificar los efectos parásitos capacitivos e inductivos debidos a las pistas en una tarjeta de circuito impreso de una cara, se ha utilizado un filtro pasa-bajas RC de segundo orden, ampliamente conocido y estudiado, como el que se describe a continuación en la figura 5 a.

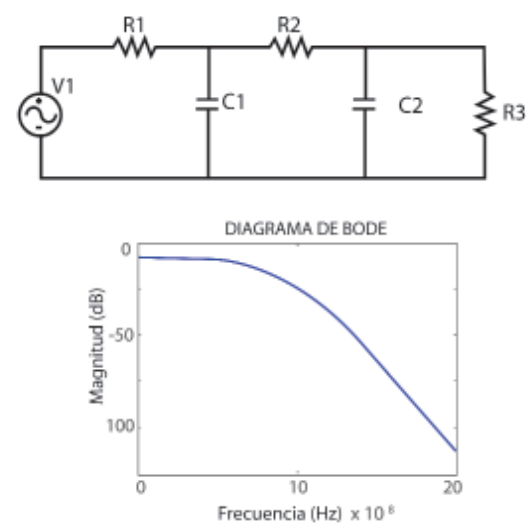

Figura 5. Filtro RC ideal a) Circuito equivalente, b) Respuesta en frecuencia
De la figura $5 b$ podemos observar que los elementos $R_{2}$ $C_{1}$ y $C_{2}$ pertenecen al filtro pasa-bajas y $R_{3}$ y $R_{1}$ a las impedancias características de los equipos de medición.

En Uribe (2005), se realizó un estudio de los efectos inductivos del filtro pasa-bajas RC de segundo orden mostrado en la figura $5 \mathrm{~b}$. En la parte experimental se observó que la respuesta en frecuencia del filtro no es de la forma que se muestra en la figura $5 b$ sino que presenta efectos resonantes como se muestra en la figura 6 .

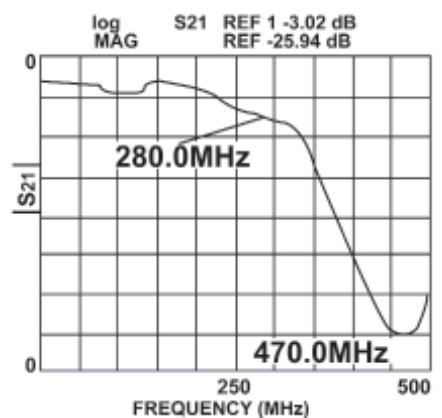

Figura 6. Forma de la respuesta en frecuencia medida de un filtro pasa-bajas RC de segundo orden, presentando efectos resonantes

Como puede observarse en la gráfica de la figura 6, existe un corrimiento en la frecuencia de corte medida con respecto a la calculada $(372.9 \mathrm{MHz})$ (Uribe et al., 2006), así como efectos resonantes debidos al intercambio de energía de un inductor con un capacitor como se muestra en la figura 7.

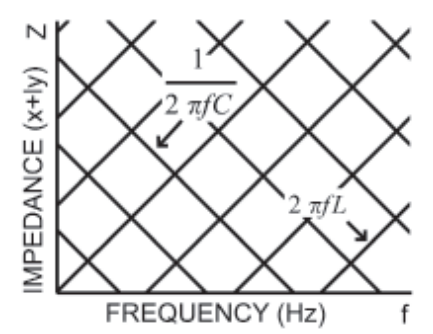

Figura 7. Líneas de impedancia de capacitores e inductores 
Por esta razón se ha propuesto una modificación del circuito eléctrico equivalente presentado en Zeeff (2003), el cual considera los efectos capacitivos mutuos que se producen entre las pistas de la TCI (Uribe, 2006), como el que se muestra en la figura 8 , el cual da una respuesta en frecuencia más próxima a la ideal. El circuito eléctrico equivalente mostrado en la figura 8 considera los efectos parásitos inductivos y capacitivos debidos a las pistas de interconexión entre los componentes empleados. Para mostrar la eficacia de éste circuito a continuación se muestra el desarrollo matemático de la función de transferencia del mismo, así como los cálculos de los valores de los capacitores e inductores que físicamente representan los efectos parásitos de las pistas de interconexión.

Determinación matemática de la función de transferencia del filtro $\mathrm{RC}$

La determinación matemática del filtro se realizó por medio de la teoría de los circuitos partiendo del circuito eléctrico equivalente que se muestra en la figura 8 . Es importante mencionar que del circuito de la figura 8:

$C_{1}=C_{\text {mutua } 1}+C_{\text {elemento1 }}$ y $\quad C_{2}=C_{\text {mutua2 }}+C_{\text {elemento2 }}$

donde

$C_{\text {elemento }}=$ valor del capacitor de montaje superficial con valor de $8.6 \mathrm{pF}$,

$C_{\text {mutua }}=$ capacitancia formada entre las pistas paralelas correspondientes a cada malla.

Los elementos que componen el filtro $\mathrm{RC}$ son dos capacitores del mismo valor denominados como $C_{\text {elemento }}$ y $R_{2}$ es la resistencia de valor igual a $10 \mathrm{Ohms}$. Los elementos $R_{1}$ y $R_{3}$ son las resistencias que presentan las impedancias características de entrada de los equipos de medición, $L_{4}$ y $L_{5}$ son las inductancias mutuas del circuito y $L_{1}, L_{2}$ y $L_{3}$ corresponden a las inductancias propias del circuito.

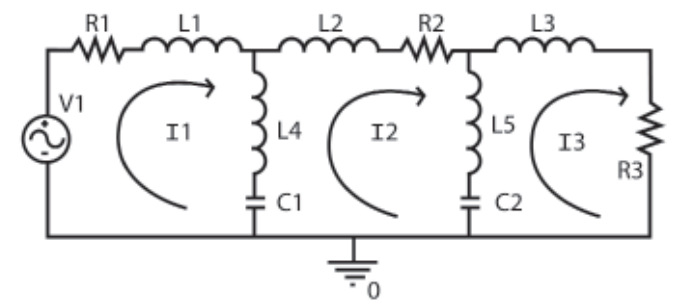

Figura 8. Circuito propuesto con elementos capacitivos e inductivos
La variación del circuito de la figura 5 respecto al de la figura 8, representa los efectos parásitos por los acoplamientos de las pistas de las TCI. Fue necesario determinar el valor de los inductores y capacitores, así como graficar su respuesta en frecuencia. La función de transferencia para el circuito propuesto de la figura 8 es:

$$
\begin{aligned}
& \frac{V_{O}}{V_{i}}=\frac{{ }_{5}^{4} R_{3} C_{1} C_{2} L_{4} L_{5}+{ }_{5}^{2} R_{3} \int C_{2} L_{5}+C_{1} L_{4} I+R_{3}}{\left(\begin{array}{l}
C_{1} C_{2} L_{1} L_{2} L_{3}+C_{1} C_{2} L_{1} L_{2} L_{5}+C_{1} C_{2} L_{1} L_{3} L_{4}+C_{1} C_{2} L_{1} L_{4} L_{5}+C_{1} C_{2} L_{1} L_{3} L_{5}+C_{1} C_{2} L_{2} L_{3} L_{4} \\
C_{1} C_{2} L_{2} L_{4} L_{5}+C_{1} C_{2} L_{3} L_{4} L_{5}
\end{array}\right)} \\
& \left(R_{1} C_{1} C_{2} L_{2} L_{3}+R_{1} C_{1} C_{2} L_{2} L_{5}+R_{1} C_{1} C_{2} L_{3} L_{4}+R_{1} C_{1} C_{2} L_{4} L_{5}+R_{1} C_{1} C_{2} L_{3} L_{5}+R_{2} C_{1} C_{2} L_{1} L_{3}+\right. \\
& +_{5^{4}}\left(\begin{array}{l}
R_{2} C_{1} C_{2} L_{1} L_{3}+R_{3} C_{1} C_{2} L_{1} L_{2}+R_{3} C_{1} C_{2} L_{1} L_{4}+R_{3} C_{1} C_{2} L_{1} L_{5}+R_{2} C_{1} C_{2} L_{3} L_{4}+R_{2} C_{1} C_{2} L_{4} L_{5}+ \\
R_{2} R_{3} C_{1} C_{2} L_{4}+R_{3} C_{1} C_{2} L_{4} L_{5}
\end{array}\right) \\
& \left(R_{1} R_{2} C_{1} C_{2} L_{3}+R_{1} R_{2} C_{1} C_{2} L_{5}+R_{1} R_{3} C_{1} C_{2} L_{2}+R_{1} R_{3} C_{1} C_{2} L_{4}+R_{1} R_{3} C_{1} C_{2} L_{5}+C_{2} L_{2} L_{3}+\right. \\
& ++_{5^{3}} C_{2} L_{2} L_{5}+C_{2} L_{3} L_{5}+R_{2} R_{3} C_{1} C_{2} L_{1}+C_{2} L_{1} L_{3}+C_{2} L_{1} L_{5}+C_{1} L_{1} L_{3}+C_{1} L_{1} L_{2}+C_{1} L_{1} L_{4}+ \\
& R_{2} R_{3} C_{1} C_{2} L_{4}+C_{1} L_{3} L_{4}+C_{1} L_{2} L_{4} \\
& +_{5^{2}}\left(\begin{array}{l}
R_{1} R_{2} R_{3} C_{1} C_{2}+R_{1} C_{2} L_{3}+R_{1} C_{2} L_{5}+R_{1} C_{1} L_{3}+R_{1} C_{1} L_{2}+R_{1} C_{1} L_{4}+R_{2} C_{2} L_{3}+R_{2} C_{2} L_{5}+ \\
R_{3} C_{2} L_{2}+R_{3} C_{2} L_{5}+R_{2} C_{1} L_{1}+R_{3} C_{2} L_{1}+R_{3} C_{1} L_{1}+R_{2} C_{1} L_{4}+R_{3} C_{1} L_{4}
\end{array}\right) \\
& ++_{5}\left(R_{1} R_{2} C_{1}+R_{1} R_{3} C_{2}+R_{1} R_{3} C_{1}+R_{2} R_{3} C_{2}+L_{1}+L_{2}+L_{3}\right)+ \\
& \left(R_{1}+R_{2}+R_{3}\right)
\end{aligned}
$$

Tarjetas de circuito impreso construidas

Se construyeron diferentes filtros, como se muestra en la figura 9. La diferencia entre ellos fue la variación del tamaño de la geometría y la variación del ancho de las pistas, como se describe a continuación:

Área de $3 \mathrm{~cm} \times 3 \mathrm{~cm}$ cada malla, con pistas de ancho $\mathrm{w}=1 \mathrm{~mm}$

Área de $3 \mathrm{~cm} \times 3 \mathrm{~cm}$ cada malla, con pistas de ancho $\mathrm{w}=0.5 \mathrm{~mm}$

Área de $2 \mathrm{~cm} \times 2 \mathrm{~cm}$ cada malla, con pistas de ancho $\mathrm{w}=1 \mathrm{~mm}$

Área de $2 \mathrm{~cm} \times 2 \mathrm{~cm}$ cada malla, con pistas de ancho $\mathrm{w}=0.5 \mathrm{~mm}$

Área de $1 \mathrm{~cm} \times 1 \mathrm{~cm}$ cada malla, con pistas de ancho $\mathrm{w}=1 \mathrm{~mm}$

Área de $1 \mathrm{~cm} \times 1 \mathrm{~cm}$ cada malla, con pistas de ancho $\mathrm{w}=0.5 \mathrm{~mm}$

Área de $0.5 \mathrm{~cm} \times 0.5 \mathrm{~cm}$ cada malla, con pistas de ancho $\mathrm{w}=1 \mathrm{~mm}$

Área de $0.5 \mathrm{~cm} \times 0.5 \mathrm{~cm}$ cada malla, con pistas de ancho $\mathrm{w}=0.5 \mathrm{~mm}$

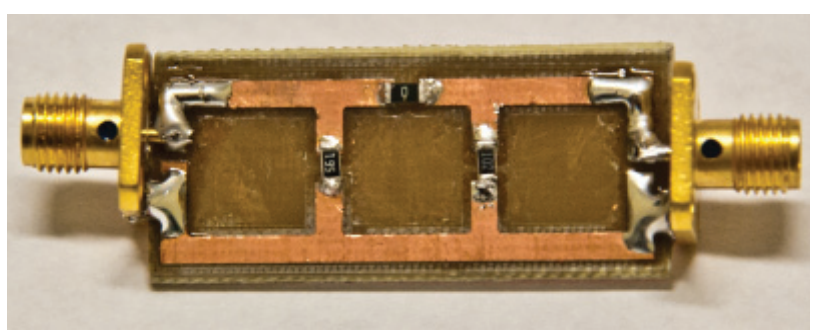

Figura 9. Topología del filtro RC de segundo orden utilizado 
Los valores de elementos usados en los filtros construidos son los siguientes:

1. $C_{1}=$ capacitor de $8.6 \mathrm{pF}$ (valor medido),

2. $C_{2}=$ capacitor de $8.6 \mathrm{pF}$ (valor medido),

3. $R_{1}=10 \mathrm{ohms}$ (valor medido).

La caracterización de los elementos fue hecha utilizando un analizador de redes vectorial.

\section{Mediciones experimentales}

Las mediciones experimentales para los filtros implementados en las diversas geometrías de TCI, descritas en la sección de desarrollo, se obtuvieron usando un analizador de redes vectorial, a través de la medición del parámetro $S_{21}$. A continuación se muestran las gráficas y los datos correspondientes a la frecuencia de corte $\left(F_{c}\right)$ y $\left(F_{r}\right)$ frecuencia de resonancia, (tablas 1 a 8; figuras 10 a 17$)$.

Tabla 1. Características del filtro RC con una separación entre capacitores $=3 \mathrm{~cm}$ y ancho de pistas $\mathrm{w}=1 \mathrm{~mm}$

\begin{tabular}{ccc}
\hline Geometría del filtro & $F_{c}$ & $F_{r}$ \\
\hline $\begin{array}{c}\text { Área de cada malla, } \\
3 \mathrm{~cm} \times 3 \mathrm{~cm}, \mathrm{w}=1 \mathrm{~mm}\end{array}$ & $\mathbf{1 6 4 . 7 5 M H z}$ & $452 \mathrm{MHz}$ \\
\hline
\end{tabular}

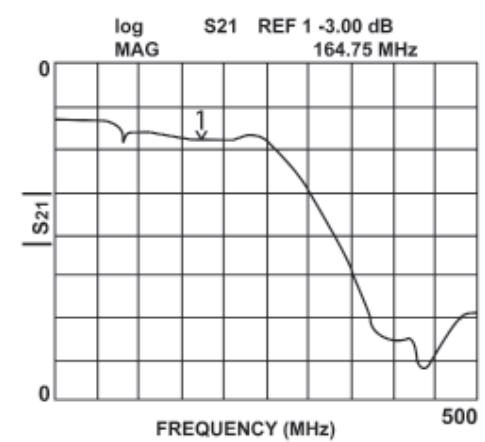

Figura 10. Respuesta en frecuencia del filtro RC de área de malla $=3 \mathrm{~cm} \times 3 \mathrm{~cm}, \mathrm{w}=1 \mathrm{~mm}$.

Tabla 2. Características del filtro RC con una separación entre capacitores $=3 \mathrm{~cm}$ y ancho de pistas $\mathrm{w}=0.5 \mathrm{~mm}$

\begin{tabular}{ccc}
\hline Geometría del filtro & $F_{c}$ & $F_{r}$ \\
\hline $\begin{array}{c}\text { Área de cada malla, } \\
3 \mathrm{~cm} \times 3 \mathrm{~cm}, \mathrm{w}=0.5 \mathrm{~mm}\end{array}$ & $\mathbf{1 3 6 \mathrm { MHz }}$ & $452 \mathrm{MHz}$ \\
\hline
\end{tabular}

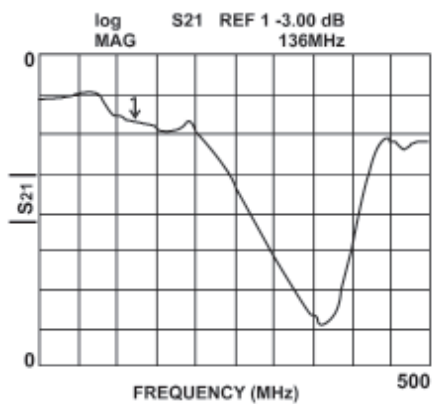

Figura 11. Respuesta en frecuencia del filtro RC de área de malla $=3 \mathrm{~cm} \times 3 \mathrm{~cm}, \mathrm{w}=0.5 \mathrm{~mm}$

Tabla 3. Características del filtro RC con una separación entre capacitores $=2 \mathrm{~cm}$ y ancho de pistas $\mathrm{w}=1 \mathrm{~mm}$

\begin{tabular}{ccc}
\hline Geometría del filtro & $F_{c}$ & $F_{r}$ \\
\hline $\begin{array}{c}\text { Área de cada malla, } \\
2 \mathrm{~cm} \times 2 \mathrm{~cm}, \mathrm{w}=1 \mathrm{~mm}\end{array}$ & $254.25 \mathrm{MHz}$ & $472.75 \mathrm{MHz}$ \\
\hline
\end{tabular}

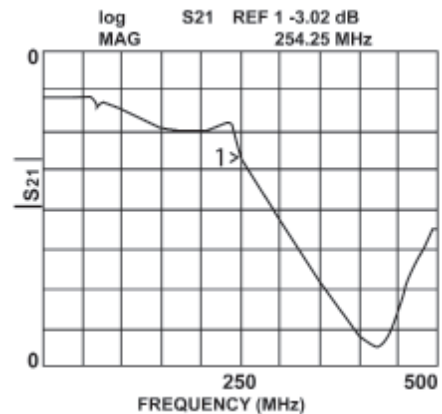

Figura 12. Respuesta en frecuencia del filtro RC de área de malla $=2 \mathrm{~cm} \times 2 \mathrm{~cm}, \mathrm{w}=1 \mathrm{~mm}$.

Tabla 4. Características del filtro RC con una separación entre capacitores $=2 \mathrm{~cm}$ y ancho de pistas $\mathrm{w}=0.5 \mathrm{~mm}$

\begin{tabular}{ccc}
\hline Geometría del filtro & $F_{c}$ & $F_{r}$ \\
\hline $\begin{array}{c}\text { Área de cada malla, } \\
2 \mathrm{~cm} \times 2 \mathrm{~cm}, \mathrm{w}=0.5 \mathrm{~mm}\end{array}$ & $207.5 \mathrm{MHz}$ & $458 \mathrm{MHz}$ \\
\hline
\end{tabular}

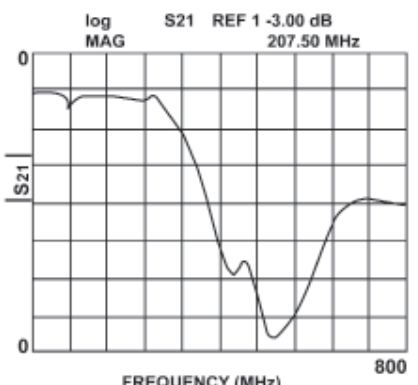

Figura 13. Respuesta en frecuencia del filtro RC de área de malla $=2 \mathrm{~cm} \times 2 \mathrm{~cm}, \mathrm{w}=0.5 \mathrm{~mm}$ 
Tabla 5 Características del filtro RC con una separación entre capacitores $=1 \mathrm{~cm}$ y ancho de pistas $\mathrm{w}=1 \mathrm{~mm}$

\begin{tabular}{ccc}
\hline Geometría del filtro & $F_{c}$ & $F_{r}$ \\
\hline Área de cada malla, & $386.37 \mathrm{MHz}$ & $717.87 \mathrm{MHz}$ \\
$1 \mathrm{~cm} \times 1 \mathrm{~cm}, \mathrm{w}=1 \mathrm{~mm}$ & & $947.00 \mathrm{GHz}$ \\
\hline
\end{tabular}

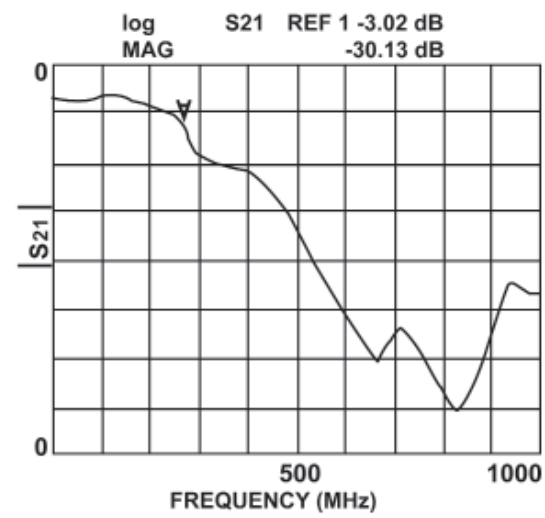

Figura 14. Respuesta en frecuencia del filtro RC de área de malla $=1 \mathrm{~cm} \times 1 \mathrm{~cm}, \mathrm{w}=1 \mathrm{~mm}$

Tabla 6. Características del filtro RC con una separación entre capacitores $=1 \mathrm{~cm}$ y ancho de pistas $\mathrm{w}=0.5 \mathrm{~mm}$

\begin{tabular}{ccc}
\hline Geometría del filtro & $F_{c}$ & $F_{r}$ \\
\hline $\begin{array}{c}\text { Área de cada malla, } \\
1 \mathrm{~cm} \times 1 \mathrm{~cm}, \mathrm{w}=0.5 \mathrm{~mm}\end{array}$ & 343.25 & $717.00 \mathrm{MHz}$ \\
\hline
\end{tabular}

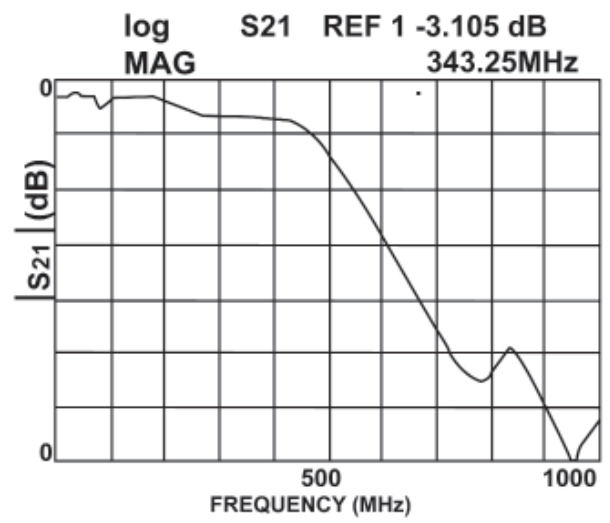

Figura 15. Respuesta en frecuencia del filtro RC de área de malla $=1 \mathrm{~cm} \times 1 \mathrm{~cm}, \mathrm{w}=0.5 \mathrm{~mm}$
Tabla 7. Características del filtro RC con una separación entre capacitores $=0.5 \mathrm{~cm}$ y ancho de pistas $\mathrm{w}=1 \mathrm{~mm}$

\begin{tabular}{ccc}
\hline Geometría del filtro & $\mathrm{F}_{\mathrm{c}}$ & $\mathrm{F}_{\mathrm{r}}$ \\
\hline Área de cada malla, & 400 & $993.0 \mathrm{MHz}$ \\
$0.5 \mathrm{~cm} \times 0.5 \mathrm{~cm}, \mathrm{w}=1 \mathrm{~mm}$ & $\mathrm{MHz}$ & $1.11 \mathrm{GHz}$ \\
\hline
\end{tabular}

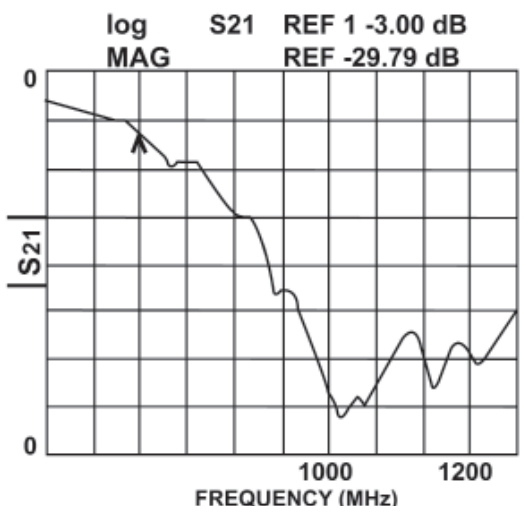

Figura 16. Respuesta en frecuencia del filtro RC de área de malla $=0.5 \mathrm{~cm} \times 0.5 \mathrm{~cm}, \mathrm{w}=1 \mathrm{~mm}$

Tabla 8. Características del filtro RC con una separación entre capacitores $=0.5 \mathrm{~cm}$ y ancho de pistas $\mathrm{w}=0.5 \mathrm{~mm}$

\begin{tabular}{ccc}
\hline Geometría del filtro & $\mathrm{F}_{\mathrm{c}}$ & $\mathrm{F}_{\mathrm{r}}$ \\
\hline Área de cada malla, & $397.85 \mathrm{MHz}$ & $\begin{array}{c}958.5 \mathrm{MHz} \\
1.09 \mathrm{GHz} \\
0.5 \mathrm{~cm} \times 0.5 \mathrm{~cm}, \mathrm{w}=0.5 \mathrm{~mm}\end{array}$ \\
\hline
\end{tabular}

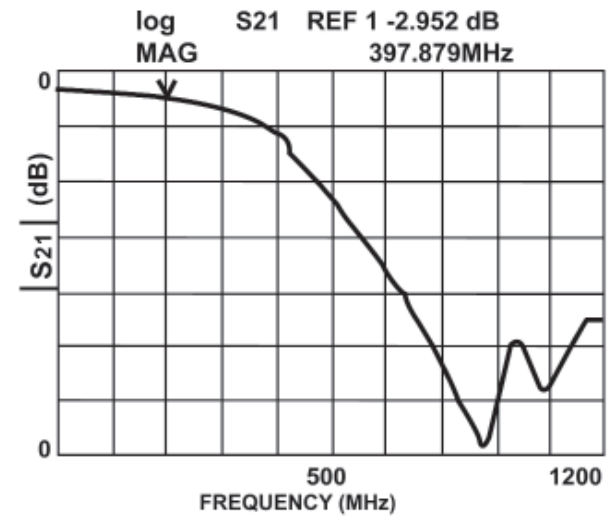

Figura 17. Respuesta en frecuencia del filtro RC de área de malla $=0.5 \mathrm{~cm} \times 0.5 \mathrm{~cm}, \mathrm{w}=0.5 \mathrm{~mm}$ 
Tabla 9. Valores de inductancias y capacitancias de cada filtro construido en $\mathrm{TCl}$

\begin{tabular}{|c|c|c|c|c|}
\hline Área de la Malla & $\begin{array}{c}L_{1} \\
L_{3} \\
{[\mathrm{nH}]}\end{array}$ & $\begin{array}{c}L_{2} \\
{[\mathrm{nH}]}\end{array}$ & $\begin{array}{c}L_{4} \\
L_{5} \\
{[\mathrm{nH}]}\end{array}$ & $\begin{array}{c}C_{m 1} \\
C_{m 2} \\
{[\mathrm{pF}]}\end{array}$ \\
\hline $\begin{array}{c}9 \mathrm{~cm}^{2} \\
(\mathrm{w}=1 \mathrm{~mm})\end{array}$ & 120 & 101.82 & 10.107 & 4.246 \\
\hline $\begin{array}{c}9 \mathrm{~cm}^{2} \\
(\mathrm{w}=0.5 \mathrm{~mm})\end{array}$ & 141.48 & 119.47 & 10.847 & 4.612 \\
\hline $\begin{array}{c}4 \mathrm{~cm}^{2} \\
(\mathrm{w}=1 \mathrm{~mm})\end{array}$ & 61.11 & 49.56 & 6.763 & 2.051 \\
\hline $\begin{array}{c}4 \mathrm{~cm}^{2} \\
(\mathrm{w}=0.5 \mathrm{~mm})\end{array}$ & 85 & 71.90 & 6.30 & 2.65 \\
\hline $\begin{array}{c}1 \mathrm{~cm}^{2} \\
(\mathrm{w}=1 \mathrm{~mm})\end{array}$ & 28.69 & 22.57 & 3.45 & 0.80 \\
\hline $\begin{array}{c}1 \mathrm{~cm}^{2} \\
(\mathrm{w}=0.5 \mathrm{~mm})\end{array}$ & 35.53 & 29.02 & 3.10 & 0.995 \\
\hline $\begin{array}{c}0.25 \mathrm{~cm}^{2} \\
(\mathrm{w}=1 \mathrm{~mm})\end{array}$ & 11.90 & 10 & 2.44 & 0.306 \\
\hline $\begin{array}{c}0.25 \mathrm{~cm}^{2} \\
(\mathrm{w}=0.5 \mathrm{~mm})\end{array}$ & 15.65 & 8.64 & 2.98 & 0.265 \\
\hline
\end{tabular}

\section{Determinación de los valores de los elementos}

Haciendo uso de las ecuaciones descritas en la secciones anteriores y el programa MatLab, se pueden obtener los valores correspondientes a las inductancias y capacitancias del filtro, que se reportan en la tabla 9.

En la tabla 9 se observa que los valores de las inductancias y capacitancias tienen un valor significante, lo cual provoca un comportamiento del filtro ligeramente diferente al esperado, como se muestra en las siguientes secciones.

\section{Simulaciones en Serenade y Matlab}

Los programas de simulación que se utilizaron fueron dos: uno desarrollado en códigos de MatLab utilizando una interfaz gráfica, la cual por medio de las longitudes físicas de las pistas y valores de los componentes utilizados calcula las capacitancias mutuas e inductancias pro-

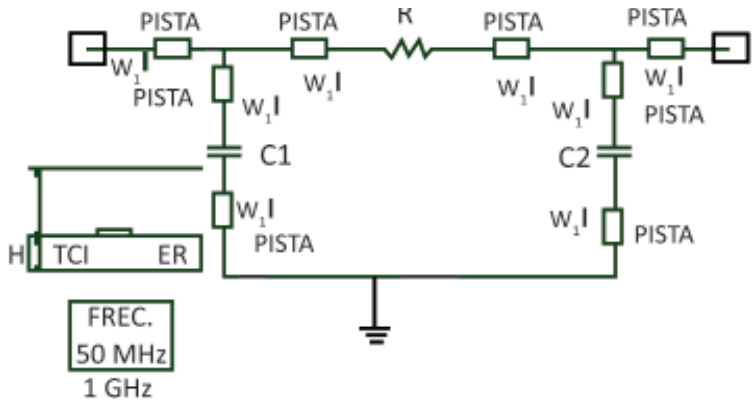

Figura 18. Circuito utilizado para la simulación en Serenade pias y mutuas del circuito eléctrico propuesto. El otro programa utilizado fue el Serenade, que utiliza segmentos de pistas de TCI donde solamente se tiene que especificar el ancho y la longitud de la pista, así como las características del material dieléctrico que utiliza la tarjeta de circuito impreso, como se muestra en la figura 18.

Como se observa en la figura 18 en el programa Serenade se debe especificar, el ancho de las pistas (w), el largo (l), así como las características de la TCI como lo son la permitividad eléctrica del dieléctrico (ER) y el grosor del mismo. Resolviendo el circuito mostrado en la figura 18 para cada uno de los casos descritos en la sección de tarjetas de circuito impreso construidas, se puede observar (Uribe, 2006) que todas las gráficas presentan el mismo comportamiento, teniendo presente una frecuencia resonante y su frecuencia de corte debido a las características propias de la TCI. En la figura 19 se muestra un ejemplo de las respuestas en frecuencia obtenidas en Uribe (2006), en este caso muestra el filtro con una geometría de $3 \mathrm{~cm} \times 3 \mathrm{~cm}$ en cada malla y con un ancho de pistas de $1 \mathrm{~mm}$, y presenta una frecuencia de corte de $155.73 \mathrm{MHz}$ y una frecuencia de resonancia a los 441.04MHz. Los datos de todas las simulaciones hechas con el programa comercial Serenade, de las diversas geometrías de filtros se presentan en la tabla 10.

La solución de las ecuaciones correspondientes al circuito que se muestra en la figura 18 se resolvieron en Uribe (2006) con el programa MatLab. La función de transferencia obtenida se muestra a continuación en la gráfica de la figura 20. En este caso, se utilizó un filtro con geometría de $3 \mathrm{~cm} \times 3 \mathrm{~cm}$.

Las demás gráficas simuladas en MatLab muestran el mismo comportamiento presentando el punto resonante, la única variación son los valores de las frecuencias de corte y de resonancia como se muestra en la tabla 10, donde se hace una comparación de los datos obtenidos en la parte experimental, así como en los programas de simulación.

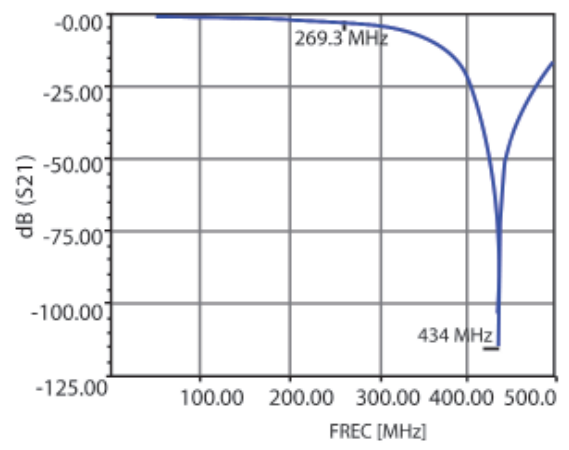

Figura 19. Respuesta en frecuencia obtenida del circuito utilizado para la simulación en el programa Serenade 


\begin{tabular}{lcccccc}
\hline & \multicolumn{2}{c}{$\begin{array}{c}\text { Datos } \\
\text { Experimentales }\end{array}$} & $\begin{array}{c}\text { Datos de } \\
\text { Simulación en } \\
\text { Matlab }\end{array}$ & \multicolumn{2}{c}{$\begin{array}{c}\text { Datos de } \\
\text { Simulación en } \\
\text { Serenade }\end{array}$} \\
\hline Mesh Area & $\begin{array}{c}F_{c} \\
{[\mathrm{MHz}]}\end{array}$ & $\begin{array}{c}F_{o} \\
{[\mathrm{MHz}]}\end{array}$ & $\begin{array}{c}F_{c} \\
{[\mathrm{MHz}]}\end{array}$ & $\begin{array}{c}F_{o} \\
{[\mathrm{MHz}]}\end{array}$ & $\begin{array}{c}F_{c} \\
{[\mathrm{MHz}]}\end{array}$ & $\begin{array}{c}F_{o} \\
{[\mathrm{MHz}]}\end{array}$ \\
$9 \mathrm{~cm}^{2}(\mathrm{w}=1 \mathrm{~mm})$ & 164.75 & 452 & $\mathbf{1 5 5 . 7 3}$ & $\mathbf{4 4 1 . 0 4}$ & 269.3 & 434 \\
$9 \mathrm{~cm}^{2}(\mathrm{w}=0.5 \mathrm{~mm})$ & 136.00 & 450 & $\mathbf{1 4 0 . 4 5}$ & $\mathbf{4 2 0 . 4 2}$ & 220.55 & 292 \\
$4 \mathrm{~cm}^{2}(\mathrm{w}=1 \mathrm{~mm})$ & 254.25 & 472 & $\mathbf{2 4 4 . 4 3}$ & $\mathbf{5 9 2 . 9 8}$ & 289 & 609 \\
$4 \mathrm{~cm}^{2}(\mathrm{w}=0.5 \mathrm{~mm})$ & 207.50 & 458 & $\mathbf{2 0 1 . 2}$ & $\mathbf{5 9 8 . 2 1}$ & 258.95 & 367 \\
$1 \mathrm{~cm}^{2}(\mathrm{w}=1 \mathrm{~mm})$ & 386.37 & 947 & $\mathbf{3 7 9 . 3 8}$ & $\mathbf{8 8 4 . 6 1}$ & 311 & 811 \\
$1 \mathrm{~cm}^{2}(\mathrm{w}=0.5 \mathrm{~mm})$ & 343.25 & 941 & $\mathbf{3 4 9 . 0 9}$ & $\mathbf{9 2 3 . 2 1}$ & 320 & 686 \\
$0.25 \mathrm{~cm}^{2}(\mathrm{w}=1 \mathrm{~mm})$ & 400.75 & 1110 & $\mathbf{4 8 3 . 5 6}$ & $\mathbf{1 0 7 9 . 8}$ & 317 & 1281 \\
$0.25 \mathrm{~cm}^{2}(\mathrm{w}=0.5 \mathrm{~mm})$ & 397.85 & 958.5 & $\mathbf{4 2 8 . 2 1}$ & $\mathbf{9 7 8 . 8 3}$ & 331 & 1398 \\
\hline
\end{tabular}

Tabla 10. Valores comparativos en forma experimental y simulada obtenidos debidos a las diferentes geometrías de filtros construidas en TCI

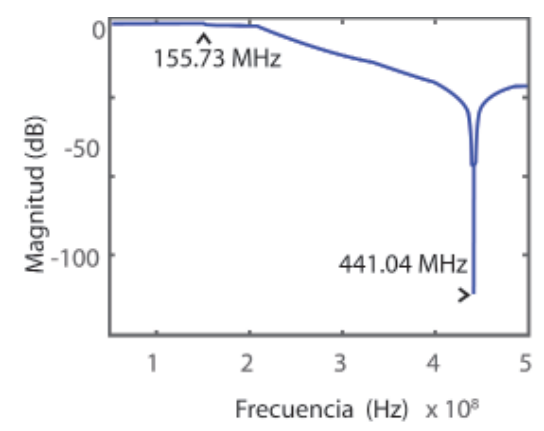

Figura 20. Respuesta en frecuencia de un filtro con geometría de $3 \mathrm{~cm} \times 3 \mathrm{~cm}$, obtenida utilizando el programa MatLab

\section{Discusión}

Se puede observar en la tabla 10 que existe una aproximación muy cercana a las mediciones experimentales, si se utiliza el programa realizado en Matlab, el cual considera los efectos inductivos y capacitivos; en cambio, el programa comercial Serenade presenta un mayor error en este caso. Es importante mencionar que la frecuencia de corte ideal es de $372.9 \mathrm{MHz}$ y las mediciones experimentales presentan una variación muy grande al cambiar las características físicas de las pistas de TCI, alejándose de la frecuencia de corte ideal causado por los efectos inductivos y capacitivos, que generalmente no se contemplan en el diseño de los sistemas electrónicos.

\section{Conclusiones}

Analizando los resultados se puede observar que si se consideran los efectos parásitos capacitivos e inductivos, que presentan las pistas de las tarjetas de circuito impre- so, se pueden hacer aproximaciones cercanas a los resultados que se presentan en la parte experimental, ya que esos efectos influyen de manera importante en el comportamiento de cualquier diseño electrónico implementado en las TCI, los cuales deben ser considerados. Es importante mencionar que el programa comercial Serenade no revela cuáles son las consideraciones que hace para obtener las gráficas en frecuencia al utilizar las pistas de circuito impreso, como se mostró en el circuito de la figura 18; sin embargo, en la tabla 10 se observa que los resultados obtenidos con este programa presentan valores diferentes a los que se obtuvieron en forma experimental, usando el programa MatLab. Por ésta razón es importante considerar los efectos parásitos descritos para obtener una mejor aproximación a la respuesta real del sistema. Este trabajo además de dar una mejor aproximación a los efectos parásitos capacitivos e inductivos que se producen debido a la geometría de las pistas utilizadas en el filtro $\pi$, nos demuestra que en cualquier diseño electrónico se deben considerar esos efectos para darle una solución antes de implementarlo en una TCI. Se puede observar que entre menor longitud y grosor de las pistas, menor inductancia propia va a existir, evitando que la frecuencia de corte se aleje de la frecuencia calculada; de igual manera si el área que se forma debido a las pistas es menor, la inductancia mutua por consecuencia va a presentar un valor inferior. La capacitancia mutua que se forma entre las pistas, es proporcional a la separación de las mismas, por lo tanto, ésta debe evitarse de forma primordial porque con el intercambio de energía de los capacitores e inductores se producen frecuencias resonantes que pueden ocurrir en un intervalo de frecuencia no deseado y hacer que el diseño electrónico sea vulnerable a presentar problemas. Con base en este 
trabajo se ha concluido de manera importante que se pueden realizar filtros utilizando las pistas de las TCI sin usar elementos resistivos ni capacitivos, solamente considerando los efectos parásitos capacitivos e inductivos variando la geometría de las pistas.

\section{Referencias}

Edwards T.C. Foundations for Microstrips Engineering, John Wiley, New York, 1981.

Gupta K.C., Garg R., Bahl I., Bhartia P. Microstirplines and Slotlines, 2ª edición, Artech House, Noorwood, MA, 1996.
Uribe-Cruz O. Estudio experimental de los efectos inductivos en las pistas de circuito impreso utilizando un filtro pasa-bajos $R C$ de dos polos, Agosto, 2005, ESIME-IPN, México.

Uribe-Cruz O., Peña-Rivero R. Inductive and Capacitive Effects in a Second Order Low-Pass Filter Constructed in PCB, 2006, 3rd International Conference on Electrical and Electronics Engineering (ICEEE 2006).

Zeeff M.T., Hubbing H.T, Van-Doren P.T. Analysis of Simple Two-Capacitor Low-Pass Filters. IEEE Transactions on Electromagnetic Compatibility, 45(4):595-60. noviembre 2003.

\section{Semblanza de los autores}

Omar Uribe-Cruz. Obtuvo el título de ingeniero en comunicaciones y electrónica en el año 2004 por la Escuela Superior de Ingeniería Mecánica y Eléctrica Unidad Zacatenco del Instituto Politécnico Nacional. En 2006, obtuvo el grado de maestro en ciencias en ingeniería electrónica en la misma institución, donde mostró un alto desempeño académico obteniendo menciones honoríficas en sus estudios de ingeniería y también de posgrado. Obtuvo el Premio "Presea Lázaro Cárdenas 2007" en el área físico matemáticas, otorgado por el presidente de la república. Trabajó en investigaciones relacionadas con efectos parásitos capacitivos e inductivos propios y mutuos que se presentan en los lazos que se forman en las pistas de interconexión en los circuitos impresos. Actualmente trabaja como ingeniero de diseño de componentes magnéticos en la empresa transnacional Falco Electronics, ubicada en la ciudad de Mérida Yucatán.

Raúl Peña-Rivero. Obtuvo el título de ingeniero en comunicaciones y electrónica en 1993 por la Escuela Superior de Ingeniería Mecánica y Eléctrica Unidad Zacatenco del Instituto Politécnico Nacional. Obtuvo el grado de maestría en ciencias en ingeniería eléctrica en el Centro de Investigación y de Estudios Avanzados del IPN. Trabajó como investigador en el Centro Nacional de Investigación y Desarrollo Tecnológico perteneciente a la Dirección General de Institutos Tecnológicos. En 1995, se integró como profesor investigador en la Sección de Estudios de Posgrado e Investigación de la ESIME-Zacatenco del IPN. En 2006, obtuvo el grado de doctor en comunicaciones y electrónica. Su línea de investigación está relacionada con el diseño electrónico analógico y digital con enfoque hacia la compatibilidad electromagnética y el estudio del ruido de conmutación simultánea en sistemas digitales, así como en el desarrollo de filtros que minimizan sus efectos, haciendo uso de efectos parásitos que se presentan en las tarjetas de circuito impreso. Ha publicado en diversos congresos y revistas nacionales e internacionales. 